\title{
Human - Centered Approaches in Urban Analytics and Placemaking Human - Centered Approaches in Urban Analytics and Placemaking
}

\author{
Eleanna Panagoulia \\ University of California, Berkeley, USA \\ eleannapan@gmail.com
}

\begin{abstract}
Planning for resilience and enabling positive design outcomes requires combinatory methods of working with data, in order to assist decision-makers develop evidence-based methodologies and easily communicated scenarios. The staggering rise of technology integration and data-aided analysis tools in urban planning, not only facilitates our understanding of socioeconomic flux, but attempts to actively involve users as a way of creating environments that are more responsive and appropriate to their needs. This paper aims to contribute to the discourse on user involvement in design-oriented fields, in our case, urban planning, by analyzing two different approaches of participatory design.
\end{abstract}

Keywords: User-Centric Design; Open-Data; Participation; Evaluation; 'Reblock'

\section{Introduction}

Planning for resilience and enabling positive design outcomes requires combinatory methods of working with data, in order to assist decision-makers develop evidencebased methodologies and easily communicated scenarios. To accomplish this we need to bring together data and information sets from disparate and vastly divergent disciplines and sources. The staggering rise of technology integration and data-aided analysis tools in urban planning, not only facilitates our understanding of socio-economic flux, but attempts to actively involve users as a way of creating environments that are more responsive and appropriate to their needs (Sassen, 1998). This shifts our thinking towards democratic environments, where users engage designers by expressing their preferences on how an idea could become part of their lives. This paper aims to contribute to the discourse on user involvement in design-oriented fields, in our case, urban planning, by analyzing two different approaches of participatory design. The first approach addresses user participation as a research method or an analysis tool and the second, as an urban design method. The key aspect to both approaches is open data platforms, as they allow access to the intended audience, researcher or average user. Both approaches are presented through example studies that are analyzed and compared based on the type of user participation, amount of user involvement and type of context they're applied to. The two methods represent different stages of participatory design, where the first focuses on the integration of human perspective in neighborhood evaluation and the other on active, contextualized user participation in placemaking and neighborhood reformation. Both processes address human perception as an effective means in capturing the dynamics of space, as well as a mean to drive the change itself. User participation is the agency upon which local resilience is formed, by balancing the power between stakeholders and community members. We support that user-centric approaches improve society well-being, user satisfaction, towards more democratic and sustainable urban environments.

\section{Participatory Design}

In order to improve policymaking and the health of communities, collaborations often extend beyond the level of academic research, to that of the user level. Recent research suggests that researchers create more innovative concepts when taking advantage of user input than working purely with existing data sets. Humans are positioned as the major contributors to changing environments (Jacobs, 1961); therefore human factor should be addressed and included when conceptualizing urban analysis methodologies. This approach has a political dimension of user empowerment and democratization and it is called participatory design approach. Participatory approaches link together all stakeholders (e.g. employees, researchers, customers, citizens, end users), in an attempt to improve human wellbeing, user satisfaction, accessibility and sustainability._As participatory processes are more and more supported by information technology, this enables both sides, users and researches, to understand and collect diverse knowledge e.g., opinions, ideas, objectives, statements etc., however it increases the complexity and the handling of information when it comes to decision-making. A participatory process involves the side of the researcher or organizer and the side of the participants. In this paper we present two different directions of the above relationship: indirect user participation and direct user participation. In the first case the users seek no personal interest in the process, however they state their opinion regarding a real matter, which is proven useful in understanding urban dynamics. This process involves two stages that depict different processes. The results are then combined in a series of maps. The second case is a deliberate process in which the interested party (citizens) is 
involved in the policy making towards the satisfaction of their needs. The process involves the construction of a digital platform that is user driven. This approach builds upon participatory action research by moving beyond participants' involvement and producing solutions to problems rather than documenting the results as a resource database. Further stages may then focus on community brainstorming, modeling and prototyping, and implementation in community spaces.

\section{Challenges of Central Urbanism Methodologies}

Urbanism during the $20^{\text {th }}$ and beginning of $21^{\text {st }}$ century was formed by large-scale centrally planned developments. A new generation of researchers has been deriving evidence-based rules for urbanism, which benefits from user participation (Salingaros, 1977). These rules replace outdated working assumptions that have created dysfunctional urban conditions. Recent methodologies in urban research validate human scale urbanism and collaborative approaches. In order to provide a better understanding of the contradictory approaches, we will list some of the main challenges of centralized urbanism.

a. Centrally- planned urbanism refers mainly to the broad picture of the urban environment; as a result, it does not address local details adequately. Centralized, top down approaches do not derive in resilient conditions as they usually favor certain economical interests. In contradiction to bottom up approaches, central-based urbanism does not rely on evidence-based methodologies and therefore it doesn't involve user participation.

b. In addition to the above, centralized approaches involve moneyoriented developments, which do not respond to local citizens needs; in fact they usually undermine them. As they largely follow the dictates of social and economic elites, they are based around uneven development, and exclusion, increasing economic segregation.

c. Citizens are often a resource of small-scale ideas that could improve the livability of their immediate environment. However, it is hard for local people to coordinate and produce visualized results that they could communicate with the authorities. Even so, such proposals are likely to be discarded as they do not represent the stakeholders' benefits and moreover, large-scale developers make it impossible for citizens to have any influence in urban development.

d. Centrally planned urbanism is based on limited data sets and assumptions, which fail to address cities as arrays of social complex relations. Such assumptions engendered vehicular domination over walkability, maximized urban density and homogenized urban districts all at the expense of residents' quality of life. It appears that there is hardly any empirical data or residents input that provide insight into most central based master plan developments.

Moving beyond the form-oriented framework of centrally based urbanism, we should also refer to certain challenges that the participatory approach entails.

\section{Challenges of Participatory Design}

The growing desire of involving participants in the process represents certain challenges that need to be addressed for successful decision-making (Sassen, 1998). The main Building user participation systems, in response to the complexity requires a combination of data, which is fit for use and decision support tools.

a. Complex data inputs. User data inputs are usually complicated data types. For example, natural language text, descriptions, sketches are a challenge for computers to interpret and also for researchers to translate them into a binary or measurable form. This type of data is also difficult to store, categorize and visualize in a proper way for future interpretation.

b. The above process is mainly a manual analysis and potential knowledge that can be drawn from the data remains obscured.

c. Ensuring that the user understands the request and is able to provide useful feedback. Abstract requests could result in user distraction, which can complicate the feedback data previously described in the first point.

Based on the above, opening a channel for sharing knowledge and opinions is not necessarily sufficient for building a system that takes the most advantage of user input. The objective is to achieve a balanced relationship between extensive information and clarity, in order to ensure that all the data and their interconnections are handled to their entirety. We need to build human - computer interaction in a way that it facilitates user orientation and comprehension of the framework, defines the scopes of the user and the researcher and translates the user input into a quantifiable entity. Therefore, we refer to a software workflow/application that ingrates user input in a form of binary data that can be easily quantified, categorized and visualized. To avoid oversimplification of the process, the insight of the researcher is crucial, in order to extract valuable, subjective information in a simple format.

\section{Example 1 - Urban Analytics though Crowdsourcing Methodologies}

The first example is a mapping process of the gentrification rate and livability levels in Oakland, San Francisco Bay Area. The data collection involves two methods; the first method the data resources derive from open data platforms (data that is freely accessible), such as Google API, Google Places and collective, open-data platforms where users post all kinds of requests (sell and buy, real estate etc.), such as "craigslist.org", while the second uses human perception and subjectivity as a qualitative source of data that can unveil qualities that could not appear otherwise.

\section{Method 1}

The database is articulated by tracing certain populations and services categories that reflect activity and flux of the built environment. The targeted data sets involve artists and their recent activity in Oakland, as well as crime reports from 2010 to 2013 (Figure 1). The data accumulation derives from open data platforms by defining an equivalent keyword query. The artist population is considered as the frontline of gentrification (Freeman, 2004); therefore tracing their activity would provide useful insight. We argue that for the artist community particularly, this data source describes effectively the activity of this group, as most of the people are freelancers or unemployed, however they actively pursue real estate for their studio or advertise artwork exhibitions etc. This activity 


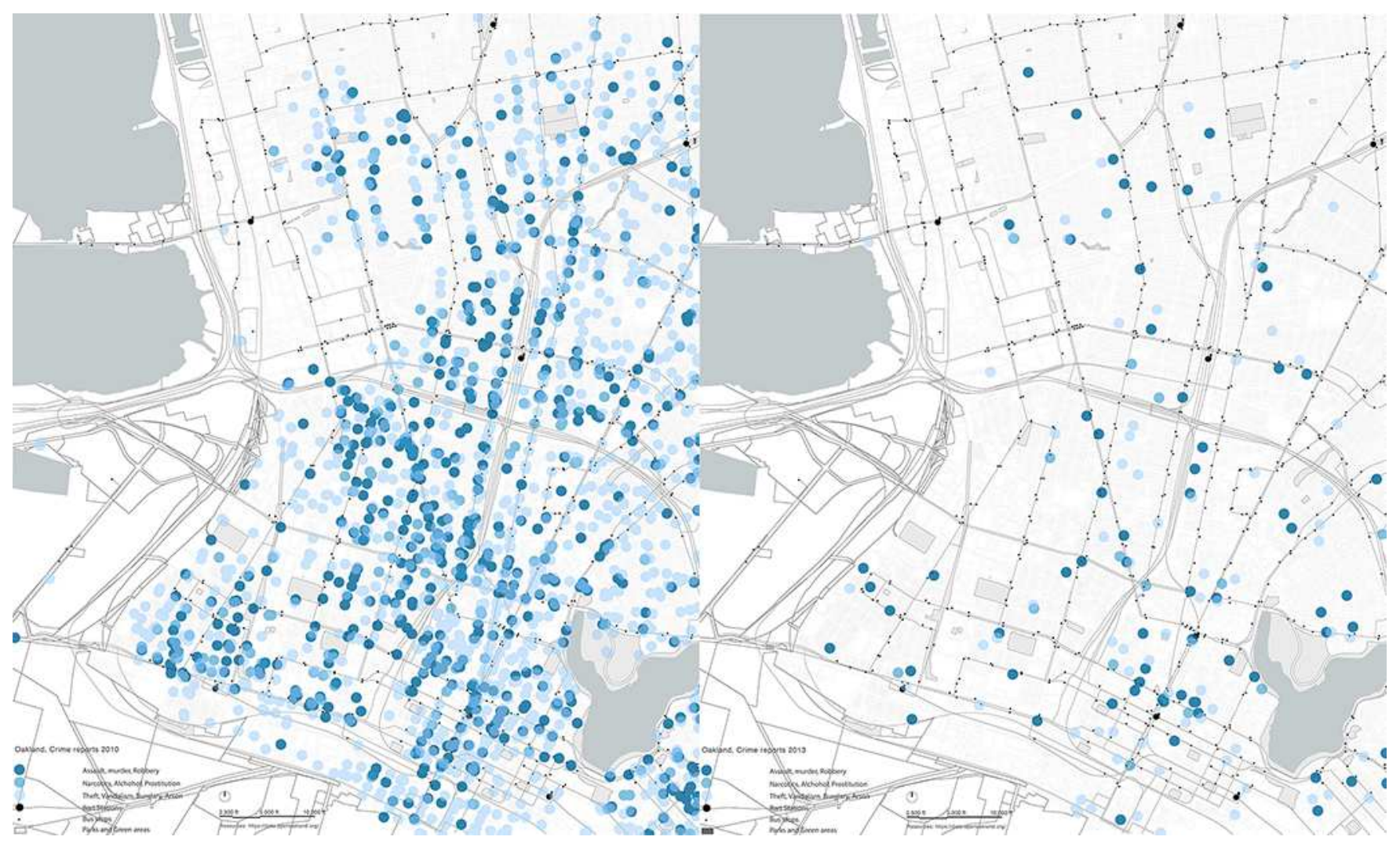

Figure 1: Oakland crime reports, 2010 left, 2013 right.

would be completely masked by a Census data analysis, however it is revealed at this stage of the process, since Google places and "craigslist.org" allow for every request is geo-located. In detail about the method itself, using Google API and "craigslist.org", we performed multiple requests at a daily basis, in order to collect all the necessary data. The keyword queries were related to temporal requests and offers regarding real estate for artists' studios, gallery spaces, events, artists' resources, artwork sale, exhibitions, FAQ etc. The second set includes crime reports posted from civilians for the years 2010 and 2013, depicting a significant decrease in reported crimes during that period. The data accumulated was formatted in .csv format and visualized as nodes on the same context.

\section{Method 2}

The second method involves a human based approach, as a crowdsourcing process. In order to allocate a group of people for crowd sourcing, we utilized a human-based outsourcing platform called Amazon Mechanical Turk. Amazon Mechanical Turk is a crowdsourcing Internet marketplace, operated by Amazon, enabling individuals to coordinate the use of human intelligence to perform tasks that computers are currently unable to do. It is an on-demand large sample of users that executes large assignments over a given period of time. In our case, a large group was given two different sets of questions (Figure 2). The first set targets human subjectivity, where the users were asked subjective questions in order to rate certain neighborhoods based on Google
Street View viewpoints. This research takes advantage of human subjectivity when it comes to rating an area based on personal interpretation of safety, affordability and infrastructure condition, qualities that vary significantly even among neighboring blocks, however the amount or the frequency of variation may have a significant role (Figure 3). The second set targets the collection of detail features (e.g. the presence of: expensive loft housing, abandoned buildings, industrial buildings, trees, fitness studios, contemporary, stylish cafes etc.) that are encountered in the areas of interest using the same Google Street View viewpoints. These features are time consuming to collect manually therefore; this tool is proven convenient as it succeeds in collecting this information in short time. The areas of interest are Oakland and Emeryville, which were chosen because they are transforming from a crime area into an urban, entertainment and commercial attractor point. The questions were submitted to Amazon Mechanical Turk through a template in .json format. The questions were structured in a way that the answers would be easy to process and to visualize, such as numerical (scale $1-10$ ), binary (yes/no) or choice (tick the box), while we avoided completely answers in a form of text. The received answers were in .json format so they were transformed into .csv format as in the previous method. All data layers were combined and provided the context for a more fine-grained understanding of neighborhood characteristics, conflicts and relationships that reveal the heterogeneous characteristics of the city (Corner, 1999). Mapping here is not addressed as a 


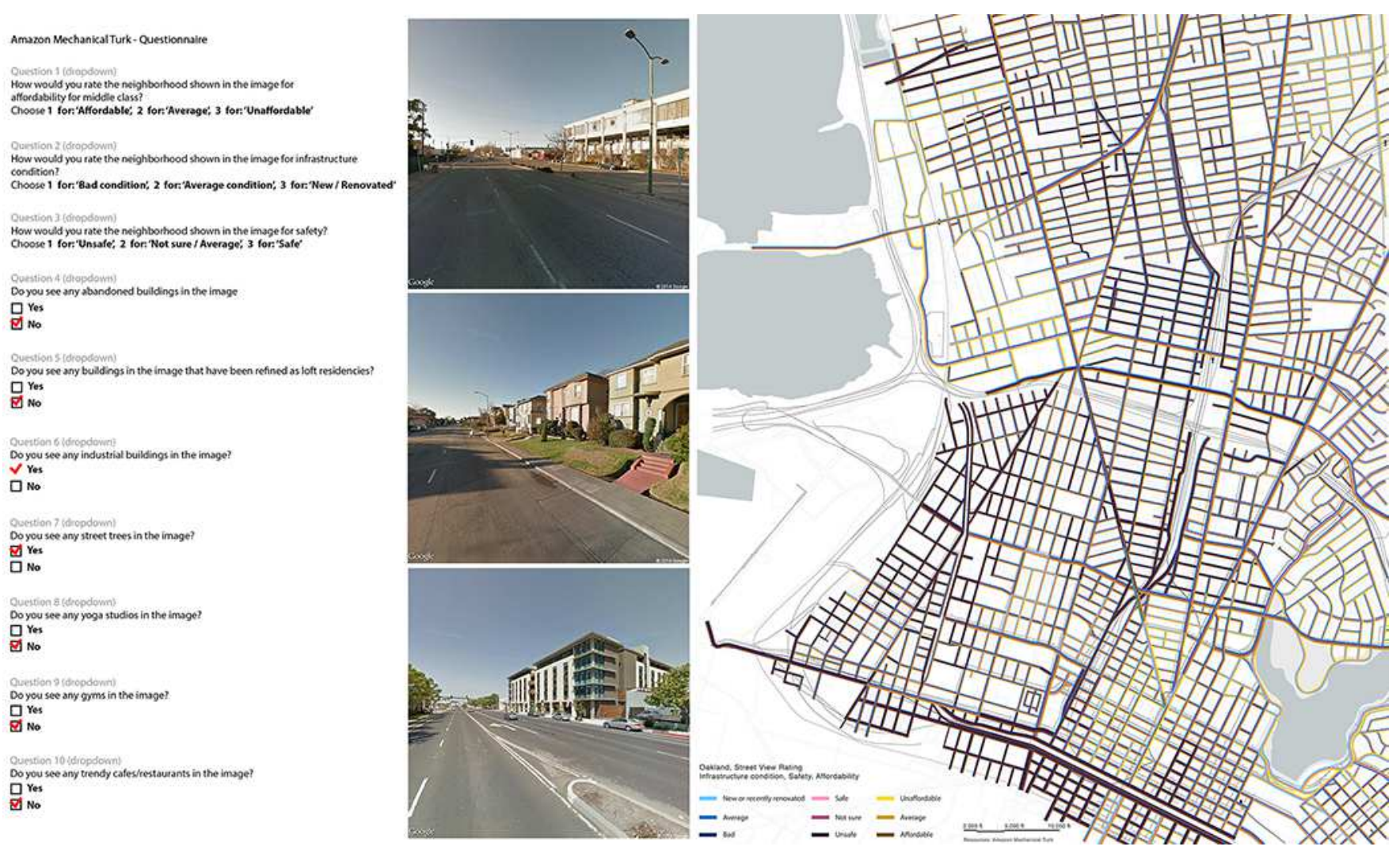

Figure 2: Oakland crime reports, 2010 left, 2013 right.

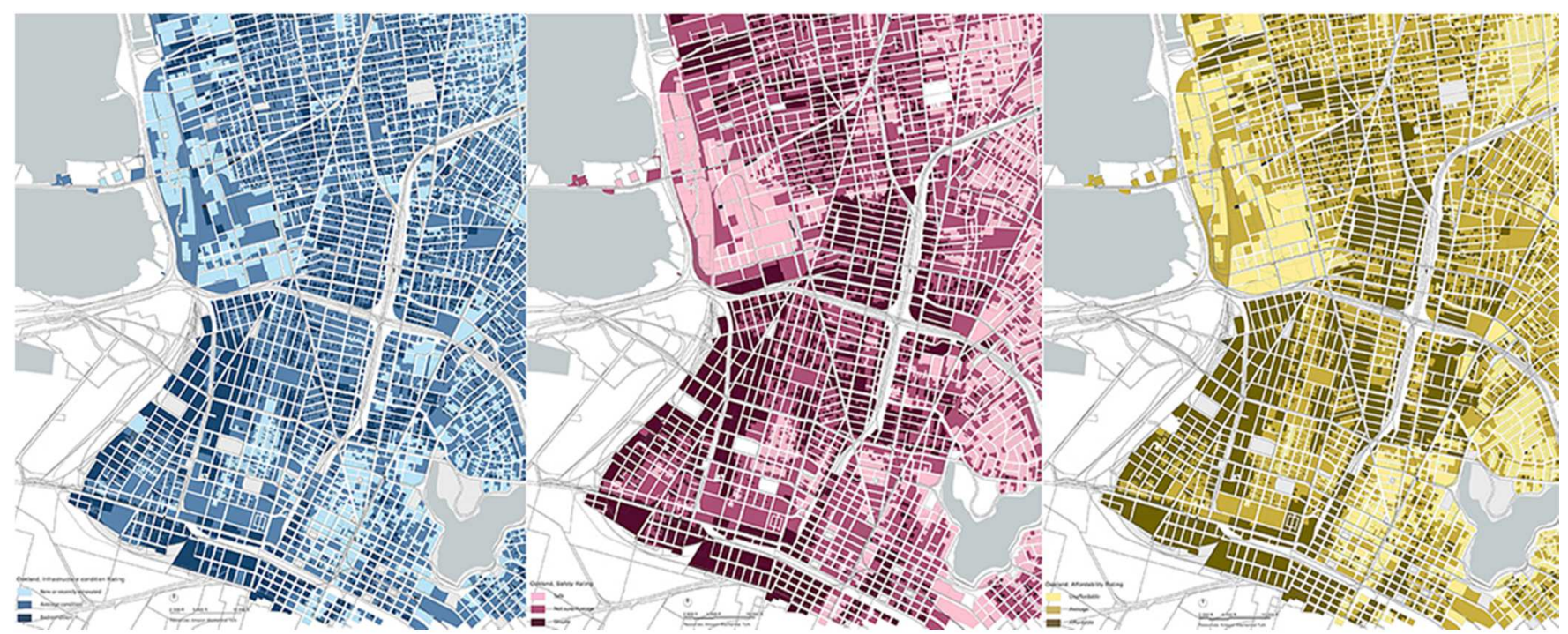

Figure 3: Oakland crime reports, 2010 left, 2013 right.

visualization tool, but also as a platform based on which we can make faster and factual assessments (De Monchaux, 2015). It is difficult to argue that either open-data or a subjective based perspective is more appropriate. Each perspective provides a different lens through which to view transition towards more or less livable environments. For example, using a human-based perspective alone may lead us to commit to something, which is entirely subjective, by ignoring holistic factors that emerge at aggregate levels. Each method presents certain advantages. The open data analysis depicts the ephemeral layer of relationships that take place in the urban environment, which is impossible to be described by authoritative data, however it is more relevant to the actual conditions, revealing user demands through open 


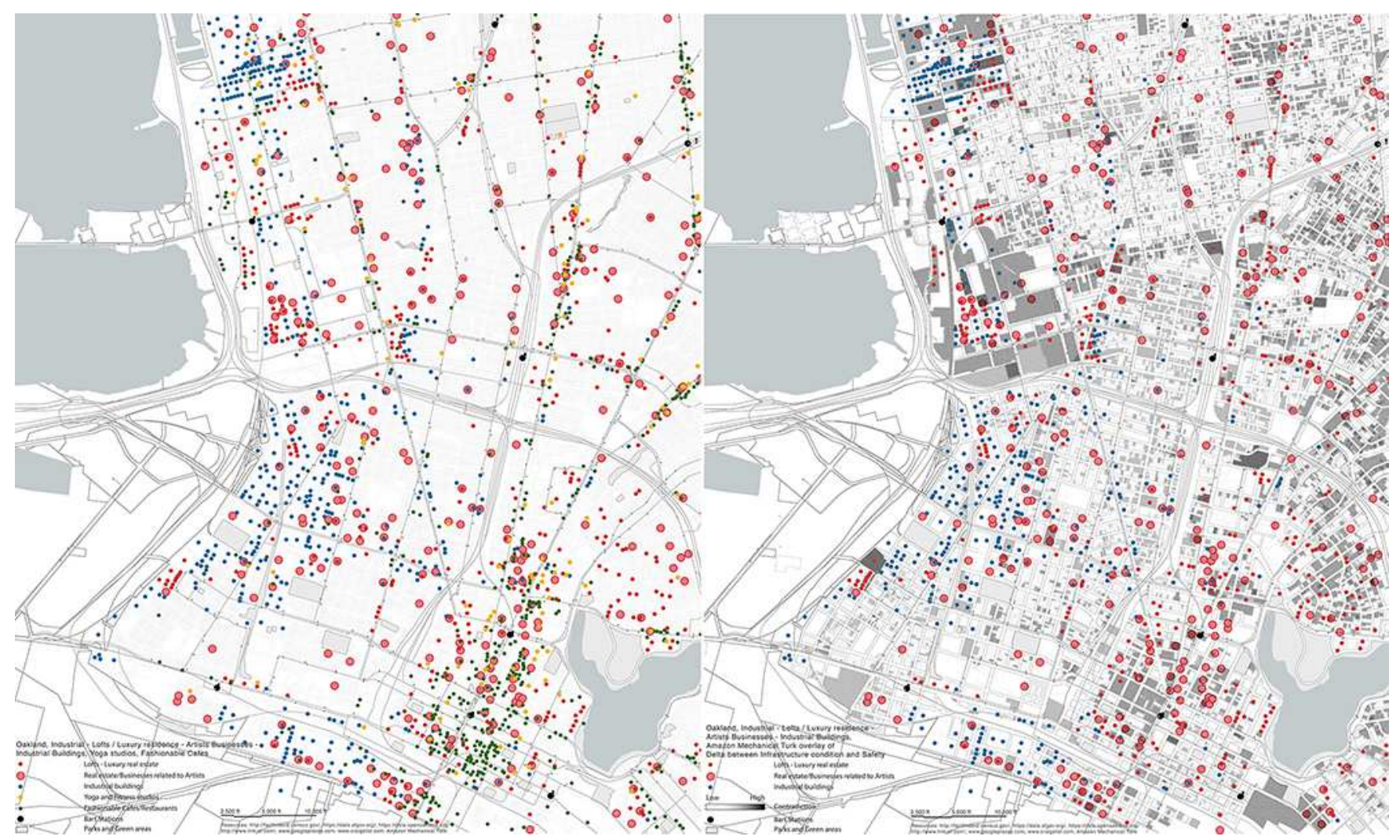

Figure 4: Oakland crime reports, 2010 left, 2013 right.

source platforms. The second method enriches the process with cultural inputs are captured as data and user personal feedback about ranking the environment of a neighborhood as it currently stands. This example has been employing evidence-based rules for urbanism, using user input that helps develop human-scale urbanism (Figure 4)

\section{Example 2 - Urban Placemaking through User Input}

Moving away from the 'expert' urbanist model, which determines the form and functionality of the built environment based on central rules, we argue that engagement with democratic participation can lead to more sustainable and resilient built environments. 'Openreblock' platform is an Open-ended approach to social justice that offers to the users active participation and opportunities to reform their immediate environment (Figure 5). The idea of the tool is that citizens have the right to affect the design of their local environment and have access to an open source methodology for doing so. As urban planning should be understood as a communicative, pragmatic, social practice, this tool facilitates intercultural dialogue and implementation. Open reblock enables users to reorganize slum communities that lack significant public infrastructure, such as access to a public street. Funded through OpenIDEO, it's the product of major research collaboration by the Santa Fe Institute, Sam Houston State University, UC Berkeley, and Shack/Slum Dwellers International, a global network of community-based organizations representing the urban poor. The tool requires user input, in order to operate and uses an algorithm to identify the least disruptive reorganization of a cluster of slum blocks so that each parcel gets access to a street. The input required is a map of the properties in the community. The design system is articulated by specific Front-End and Back. End processes (Figure 6). The front-end processes are related to the display of the website based on user demand and the back-end are related to the background processes needed for the calculation. As calculations demand a large amount of time to complete, 'Celery' has been used to queue the tasks. In order to make the process of the calculation interactive, the steps are being displayed during the calculation, so the user can spot the new paths that are being generated. The user can access and download intermediate steps of the process. The algorithm estimates the location of existing paths and associated construction costs for new streets, making discussion and comparison of alternative plans easy. It produces a new map that allows each home or workplace to have an address and to obtain urban services (Figure 7). Residents can adjust the tool to their needs by prioritizing processes and use the outcome as an alternative proposal for future re-planning, in order to oppress the local government to consider their proposal. Users can optimize the process based on their priorities, such as cost minimization, exclusion of certain paths from the calculation, because they clash with landmarks and width of the new road network for circulation convenience. This allowance for 
SIGraDi 2017, XXI Congreso de la Sociedad Ibero-americana de Gráfica Digital 22 -24, November, 2017 - Concepción, Chile.

customization is key for local resilience to climate change and socioeconomic development.

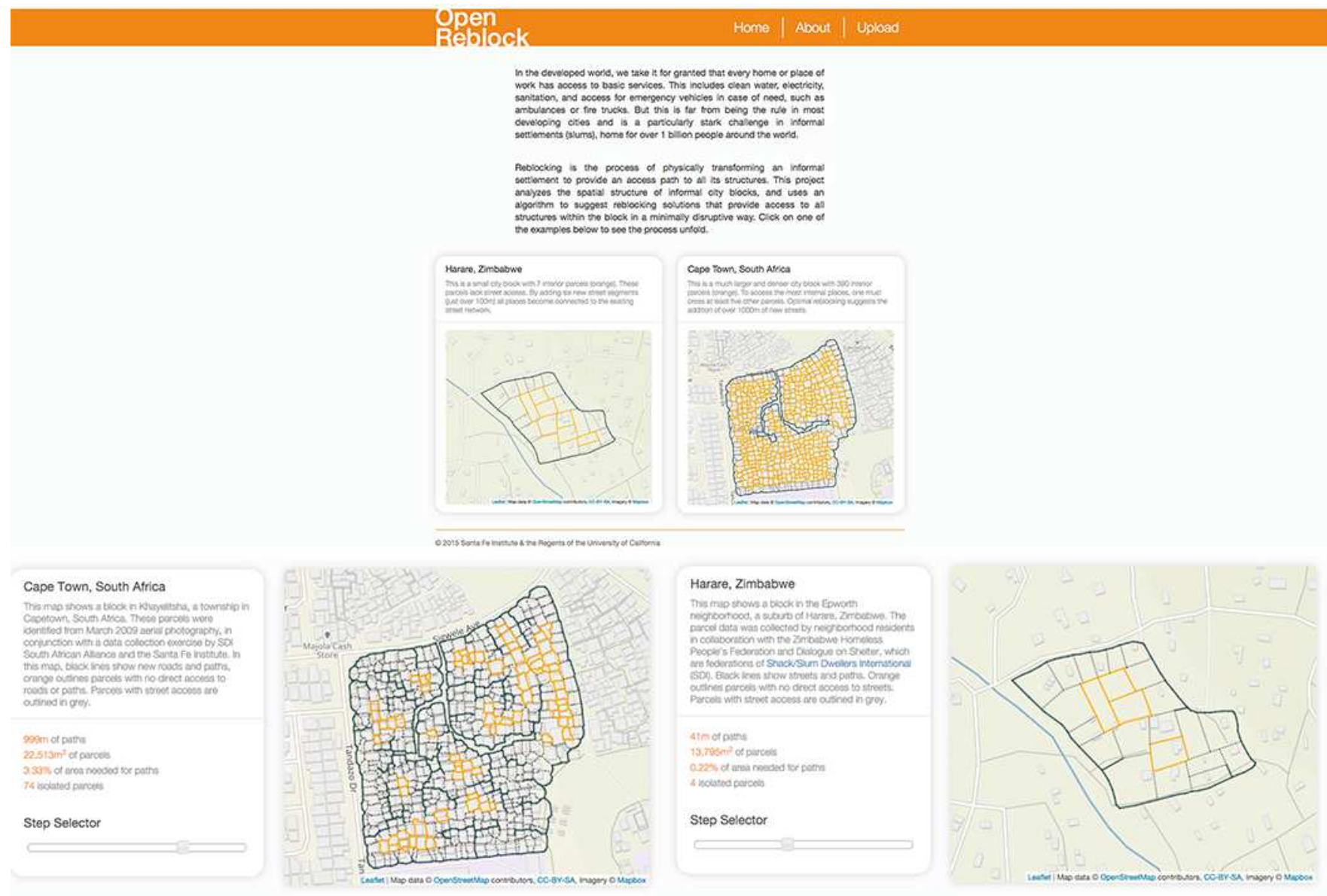

Figure 5: Openreblock: Website main page, Interface, Graphics

Front End

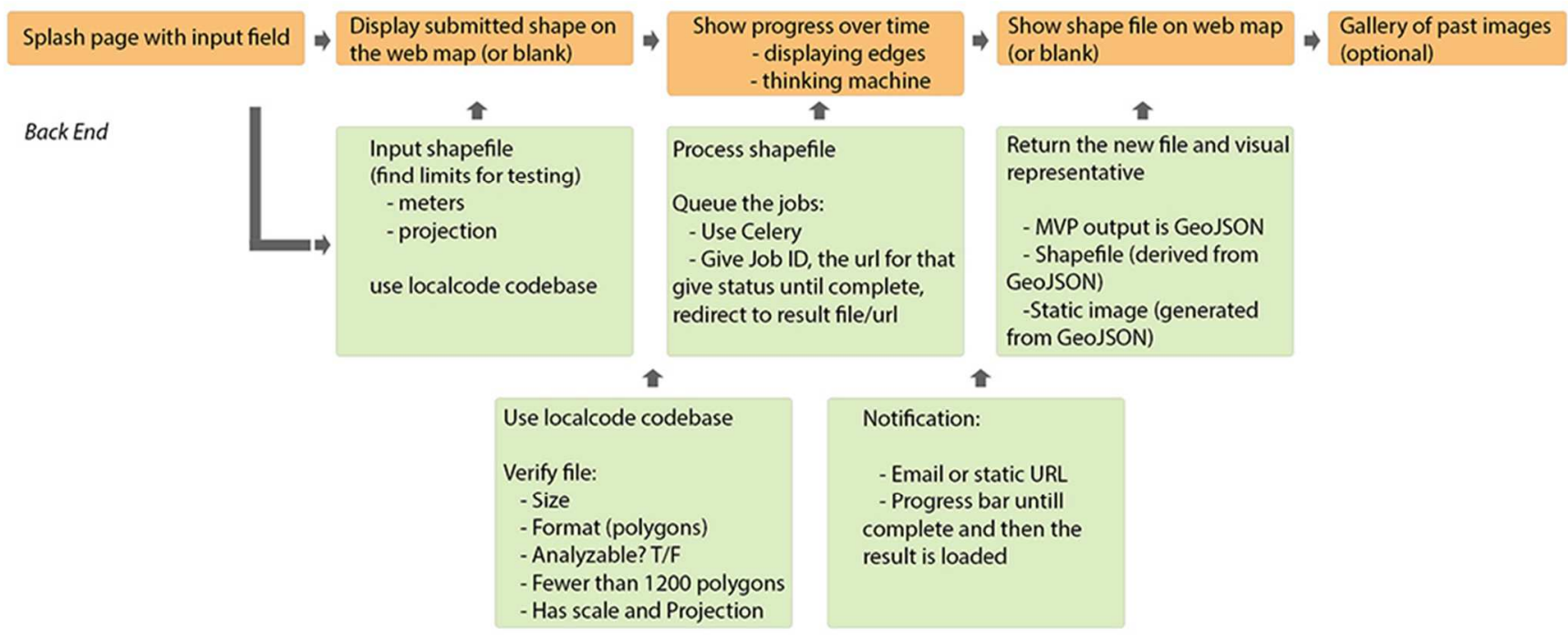




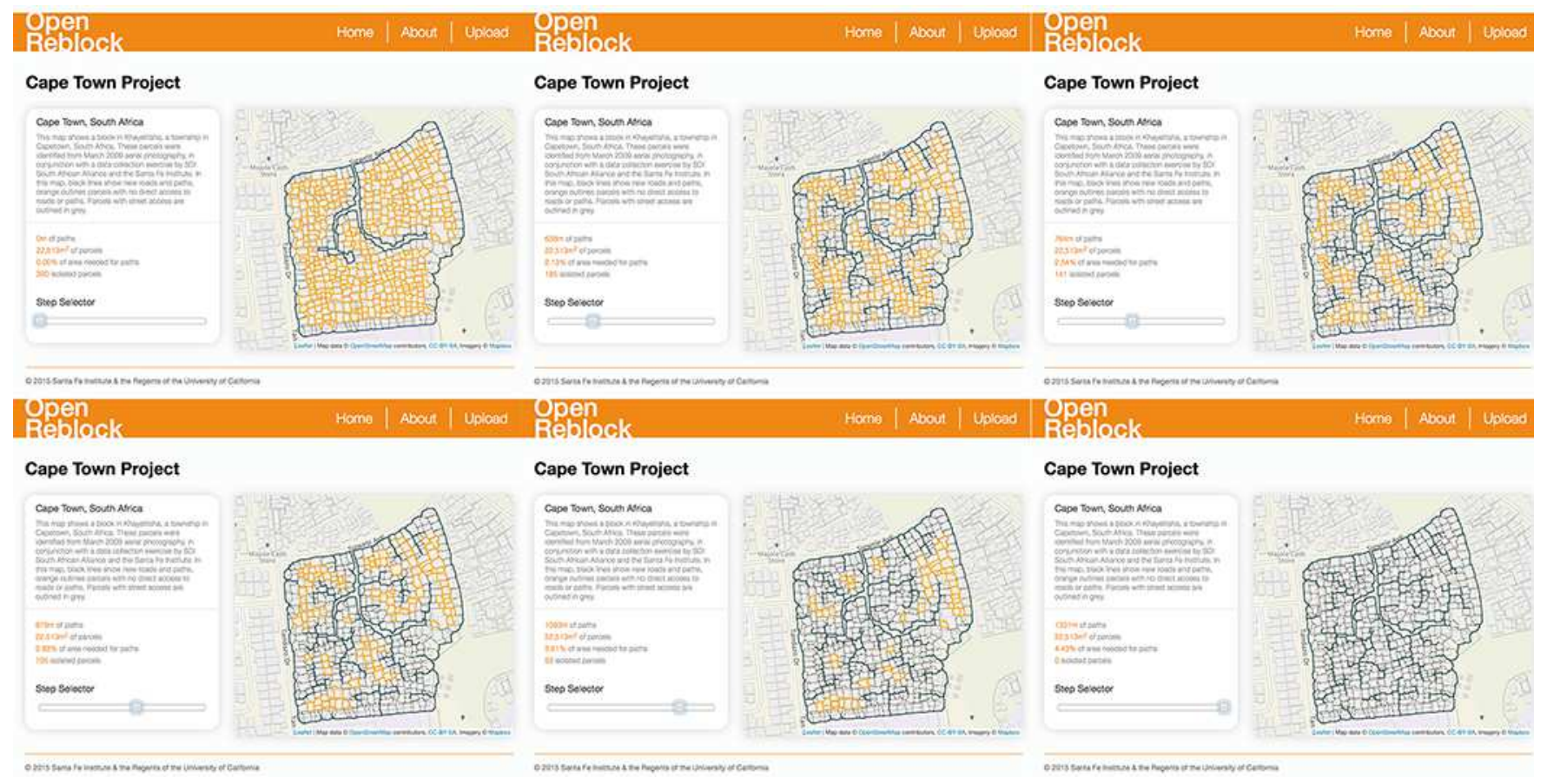

Figure 7: Cape Town example. Process of 'reblocking'. New road network is gradually formed.

\section{Discussion}

In regards to participation, the possibilities of digitalization should be regarded as an opportunity to accompany the social transformation towards a digital society in the information age of he $21^{\text {st }}$ century (Alexander et.al, 1977). Beyond its socio-political implications, participatory approach in urban planning aims to establish a framework for resilient towards sustainable environment that benefits both researches and citizens. From the researchers perspective, the ability to visualize and analyze peoples desires and opinions that reflect their background, allows for a culturally enhanced database that captures their common aspects and differences. This enrichment leads to a more informed decision-making and a more qualitative image of the city that reflects subjective aspects of urban planning (Batty, 2013). From the citizens perspective, the ability to reshape the urban fabric, prompts the residents to participate in its evolvement and grow conscience and care for their neighborhood. We believe that the key to improve policymaking is engaging people to collaborate and use information to become more active in society. This would be a first step towards the equalization of power between citizens and stakeholders and the collaborative constructions of urban space, as well as a step to understand the unique challenges that the city faces.

\section{Acknowledgments}

The development and design of the digital platform in the second example is a collaboration between College of
Environmental Design, UC Berkeley, Stamen and the Santa $\mathrm{Fe}$ Institute. Contributors from UC Berkeley include Nicolas de Monchaux, Carlos Sandoval Olascoaga, Wenzhe Peng, and Eleanna Panagoulia. Design by the Stamen design and technology studio. You can view the source for this site and the topology algorithm on GitHub.

\section{References}

Alexander, C., Ishikawa, S., Silverstein, M., Jacobson, M., FiksdahlKing, I., Angel, S. (1977). A Pattern Language. New York: Oxford University Press.

Batty, M. (2013). The new science of cities. Cambridge, Massachusetts: The MIT Press.

Corner, J. (1999). The Agency of Mapping: Speculation Critique and Invention. London: Reaktion Books.

De Monchaux, N.(2015). Local Code: 3659 Proposals About Data, Design, and the Nature of Cities, Princeton Architectural Press.

Freeman, L. (2004). Gentrification and Displacement: New York City in the 1990s. Journal of the American Planning Association 70, no.1.

Jacobs, J. (1961). The Death and Life of Great American Cities. New York: Vintage Books.

Salingaros, N. (2005) Principles of Urban Structure. Amsterdam: Techne Press.

Sassen, S. (1998). Globalization and its discontents. New York: New Press. 